\title{
Identification of Clock Synchronization Errors: A Behavioral Approach
}

\author{
Marek Przedwojski, Ivan Markovsky and Eric Rogers
}

\begin{abstract}
The subject area of this paper is discrete-time linear time-invariant systems composed of subsystems whose state updating is asynchronous due to the clock signal arriving with delays. This leads to the synchronization error identification problem which is to find from a trajectory of the system the order in which the subsystems' states are updated. A solution to this problem based on a direct search over all possible synchronization errors is developed together with conditions for its uniqueness.
\end{abstract}

\section{INTRODUCTION}

Classical digital signal processing and control system analysis techniques are based on the fundamental fact that all components of the overall system switch at exactly the same time instant. In particular, it is assumed that the system is driven by a single clock and that the differences in clock propagation time between the different system components are negligible. Over the last decade, many applications have emerged that routinely violate this basic assumption. Typical examples include wireless sensor networks, vehicle networks and swarms, tele-operation-systems, and distributed actuator systems.

Synchronization of different system components to a degree that would allow them to be treated as a synchronous system is either impossible or very expensive. Consequently research on the modeling, analysis and design of asynchronous systems is of critical importance with many applications awaiting applicable results and algorithms.

The problems arising from the introduction of non-ideal characteristics into applications such as those mentioned above can be grouped, at a general level, into time-variant communication delays caused by, for example, packet drop, queuing, access delay, and synchronization errors respectively. Moreover, the vast majority of the research has been in the former area and in [1] it is suggested that this is due to a focus on applications supported by wide area networks, where communication delays are commonly expected to be more critical than synchronization errors. On a local area network, however, the average communication delays can be made small enough such that synchronization errors become the critical issue.

This paper exclusively deals with synchronization errors, building on previous work in [1]-[6] on modeling and stability analysis. In particular, [1] showed that linear synchronous systems are subject to errors that result in asynchronous operation and moreover that this can be caused by even small errors. This previous work also demonstrated that classical

M. Przedwojski, I. Markovsky and E. Rogers are with the School of Electronics and Computer Science, University of Southampton, Southampton SO17 1BJ, United Kingdom (email: im,mp08r,etar@ecs.soton.ac.uk). design techniques cannot be directly applied to systems that operate asynchronously.

In applications, the clock signal that regulates the subsystems can reach them with (small) drifts and consequently the overall system operates asynchronously and this influences the system's response [3], [4]. As a starting point for analysis, it is assumed that the clock signal drifts remain constant and hence the discrete-time system remains time-invariant. The question considered in this paper is: can the synchronization error be identified from the input and the output of an asynchronously operating system [3], [6]?

In this work we assume that the data are exact, that is, noise free. A similar problem is treated in [3], however, their model is less general as it allows only a subset of the switching sequences considered here. We show how the problem can be solved by searching over all possible switching sequences. In contrast, the method of [3] determines the synchronization error only up to a shift transformation.

The paper is organized as follows. In section 2, a model is developed for asynchronous updating of the subsystems states. Then in section 3 the new estimation algorithm is developed and Section 4 considers identifiability conditions. Section 5 gives an illustrative example and Section 6 gives the main conclusions of the work reported here.

\section{BACKGROUND}

We consider discrete-time linear time-invariant systems defined by the state-space model

$$
\begin{aligned}
\boldsymbol{x}(k+1) & =\boldsymbol{A} \boldsymbol{x}(k)+\boldsymbol{B} \boldsymbol{u}(k), \\
\boldsymbol{y}(k) & =\boldsymbol{C} \boldsymbol{x}(k)+\boldsymbol{D} \boldsymbol{u}(k),
\end{aligned}
$$

where $\boldsymbol{x}(k) \in \mathbb{R}^{n}, \boldsymbol{u}(k) \in \mathbb{R}^{m}, \boldsymbol{y}(k) \in \mathbb{R}^{p}$ are the state vector, the input vector and the output vector at time index $k$ respectively. We assume that the basis of the state representation is fixed and that each state vector entry $x_{i}$ is fed by a clock with rate $T_{i}$ and the input vector is fed by a clock with rate $T_{n+1}$. The clock rates are equal but could be out of phase. The time index is incremented by one whenever the input updates. In case when there are no synchronization errors, all state variables update (i.e., new values are calculated) at the same time instant. If, however, synchronization errors are present then there could be more than one updating over a full clock period and in such a case (1) is no longer valid.

Assume that $d$ switching events (or updating) occur in one full clock period, described by the sequence $s$ of mutually disjoint subsets of indices

$$
s=\left(i_{1}, i_{2}, \ldots, i_{d}\right), \quad i_{j} \subseteq\{1, \ldots, n\}, \quad j=1, \ldots, d,
$$


The subset $i_{l}$, where $l=1, \ldots, d$, contains the indices of the state vector entries that are updated simultaneously during event $; l$, and all of these subsets satisfy

$$
p \neq r \Rightarrow i_{p} \cap i_{r}=\emptyset \text { for } p, r=1, \ldots, d,
$$

and

$$
\bigcup_{j=1}^{d} i_{j}=\{1, \ldots, n\} .
$$

Hence all the state variables are updated over a full clock period and the set of all possible sequences that describe switching events is denoted here by $\mathcal{S}$.

Now assume that $s$ defined in (2) describes the switching event pattern for a given example. Then when the $j$-th event occurs the state vector updating can be described by the statespace model

$$
\boldsymbol{x}^{j}(k)=\boldsymbol{A}_{i_{j}} \boldsymbol{x}^{j-1}(k)+\boldsymbol{B}_{i_{j}} \boldsymbol{u}(k),
$$

where $\boldsymbol{x}^{0}(k)=\boldsymbol{x}(k)$ and, using $i_{j}=\{\ldots, p, \ldots, q, \ldots\}$ as a representative example,

$$
\boldsymbol{A}_{i_{j}}=\left[\begin{array}{ccccc}
1 & 0 & \ldots & 0 & 0 \\
0 & 1 & \ldots & 0 & 0 \\
\ldots & \ldots & \ldots & \ldots & \ldots \\
a_{p 1} & a_{p 2} & \ldots & a_{p(n-1)} & a_{p n} \\
\ldots & \ldots & \ldots & \ldots & \ldots \\
a_{q 1} & a_{q 2} & \ldots & a_{q(n-1)} & a_{q n} \\
\ldots & \ldots & \ldots & \ldots & \ldots \\
0 & 0 & \ldots & 1 & 0 \\
0 & 0 & \ldots & 0 & 1
\end{array}\right]
$$

and

$$
\boldsymbol{B}_{i_{j}}=\left[\begin{array}{ccccc}
0 & 0 & \ldots & 0 & 0 \\
0 & 0 & \ldots & 0 & 0 \\
\ldots & \ldots & \ldots & \ldots & \ldots \\
b_{p 1} & b_{p 2} & \ldots & b_{p(m-1)} & b_{p m} \\
\ldots & \ldots & \ldots & \ldots & \ldots \\
b_{q 1} & b_{q 2} & \ldots & b_{q(m-1)} & b_{q m} \\
\ldots & \ldots & \ldots & \ldots & \ldots \\
0 & 0 & \ldots & 0 & 0 \\
0 & 0 & \ldots & 0 & 0
\end{array}\right] .
$$

Hence during event $j$ th only the state vector entries corresponding to $i_{j}$ are updated, and we assume that $\boldsymbol{x}(k+1)=$ $\boldsymbol{x}^{d}(k)$, so a new $n \times 1$ state vector is created after all switching events have taken place, resulting in the state-space model

$$
\begin{aligned}
\boldsymbol{x}(k+1) & =\boldsymbol{A}_{s} \boldsymbol{x}(k)+\boldsymbol{B}_{s} \boldsymbol{u}(k), \\
\boldsymbol{y}(k) & =\boldsymbol{C} \boldsymbol{x}(k)+\boldsymbol{D} \boldsymbol{u}(k),
\end{aligned}
$$

where

$$
\boldsymbol{A}_{s}=\boldsymbol{A}_{i_{d}} \cdots \boldsymbol{A}_{i_{1}}
$$

and

$$
\boldsymbol{B}_{s}=\boldsymbol{B}_{i_{d}}+\boldsymbol{A}_{i_{d}} \boldsymbol{B}_{i_{d-1}}+\cdots+\boldsymbol{A}_{i_{d}} \cdots \boldsymbol{A}_{i_{2}} \boldsymbol{B}_{i_{1}} .
$$

Example 1: Consider a 2nd order system with zero input and

$$
\boldsymbol{A}=\left[\begin{array}{ll}
a_{11} & a_{12} \\
a_{21} & a_{22}
\end{array}\right]
$$

Assuming that first entry is updated before the second one, the corresponding sequence $s$ is

$$
s=(\{1\},\{2\}) .
$$

Event 1 corresponds to $i_{1}=\{1\}$, and

$$
\begin{aligned}
{\left[\begin{array}{l}
x_{1}^{1}(k) \\
x_{2}^{1}(k)
\end{array}\right] } & =\left[\begin{array}{cc}
a_{11} & a_{12} \\
0 & 1
\end{array}\right]\left[\begin{array}{l}
x_{1}(k) \\
x_{2}(k)
\end{array}\right] \\
& =\boldsymbol{A}_{\{1\}}\left[\begin{array}{l}
x_{1}(k) \\
x_{2}(k)
\end{array}\right] .
\end{aligned}
$$

Event 2 corresponds to $i_{2}=\{2\}$, and

$$
\begin{aligned}
{\left[\begin{array}{l}
x_{1}^{2}(k) \\
x_{2}^{2}(k)
\end{array}\right] } & =\left[\begin{array}{cc}
1 & 0 \\
a_{21} & a_{22}
\end{array}\right]\left[\begin{array}{l}
x_{1}^{1}(k) \\
x_{2}^{1}(k)
\end{array}\right] \\
& =\boldsymbol{A}_{\{2\}}\left[\begin{array}{l}
x_{1}^{1}(k) \\
x_{2}^{1}(k)
\end{array}\right] .
\end{aligned}
$$

Since $\boldsymbol{x}(k+1)=\boldsymbol{x}^{2}(k)$, the state vector after one clock period is

$$
\begin{aligned}
{\left[\begin{array}{c}
x_{1}(k+1) \\
x_{2}(k+1)
\end{array}\right] } & =\left[\begin{array}{cc}
1 & 0 \\
a_{21} & a_{22}
\end{array}\right] \cdot\left[\begin{array}{cc}
a_{11} & a_{12} \\
0 & 1
\end{array}\right]\left[\begin{array}{l}
x_{1}(k) \\
x_{2}(k)
\end{array}\right] \\
& =\boldsymbol{A}_{\{2\}} \boldsymbol{A}_{\{1\}}\left[\begin{array}{l}
x_{1}(k) \\
x_{2}(k)
\end{array}\right]
\end{aligned}
$$

for $k=0,1, \ldots$

The analysis in this paper will also require the extended observability matrix for discrete linear time invariant systems described by (1), that is,

$$
\boldsymbol{O}_{k}(\boldsymbol{A}, \boldsymbol{C})=\left[\begin{array}{c}
\boldsymbol{C} \\
\boldsymbol{C A} \\
\vdots \\
\boldsymbol{C} \boldsymbol{A}^{k-1}
\end{array}\right]
$$

We also use the Toeplitz matrix constructed from (1) as

$$
\boldsymbol{T}_{k}(\boldsymbol{A}, \boldsymbol{B}, \boldsymbol{C}, \boldsymbol{D})=\left[\begin{array}{cccc}
\boldsymbol{D} & 0 & \ldots & 0 \\
\boldsymbol{C B} & \boldsymbol{D} & \ddots & \vdots \\
\vdots & \ddots & \ddots & 0 \\
\boldsymbol{C} \boldsymbol{A}^{k-2} \boldsymbol{B} & \ldots & \boldsymbol{C} \boldsymbol{B} & \boldsymbol{D}
\end{array}\right]
$$

and the augmented output and input vectors

$$
\boldsymbol{Y}_{k}=\left[\begin{array}{c}
\boldsymbol{y}(1) \\
\boldsymbol{y}(2) \\
\vdots \\
\boldsymbol{y}(k)
\end{array}\right], \quad U_{k}=\left[\begin{array}{c}
\boldsymbol{u}(1) \\
\boldsymbol{u}(2) \\
\vdots \\
\boldsymbol{u}(k)
\end{array}\right]
$$

The analysis in later sections also uses results from the behavioral approach to systems analysis, where an overview of this approach and comprehensive referencing of the literature can be found in, for example, [7]. To introduce the basic concepts required here, let $\mathbb{T}$ be the time axis and $\mathbb{W}$ the signal space associated with a given system. Then is this setting a dynamical system is defined as a triple

$$
\Sigma=(\mathbb{T}, \mathbb{W}, \mathcal{B}),
$$

where $\mathcal{B}$ denotes the behavior of the system which is a subset of $\mathbb{W}^{\mathbb{T}}$, where $\mathbb{W}^{\mathbb{T}}$ denotes the set of all maps from $\mathbb{T}$ to $\mathbb{W}$. 
Here we have $\mathbb{T}=\mathbb{N}$ and we partition $\mathbb{W}$ as $\mathbb{U} \times \mathbb{Y}$, where for $\boldsymbol{w} \in \mathbb{W}$ with corresponding partition $\boldsymbol{w}=(\boldsymbol{u}, \boldsymbol{y}), \boldsymbol{u}$ is an input and $\boldsymbol{y}$ is an output. The behavior of the system (1), that is, the set of signals that can occur in the system, can now be defined as

$$
\mathcal{B}(\boldsymbol{A}, \boldsymbol{B}, \boldsymbol{C}, \boldsymbol{D})=\left\{(\boldsymbol{u}, \boldsymbol{y}): \begin{array}{c}
\text { there exists } \boldsymbol{x} \\
\text { such that (1) holds }
\end{array}\right\} .
$$

Also

$$
\mathcal{B}(\boldsymbol{A}, \boldsymbol{B}, \boldsymbol{C}, \boldsymbol{D})=\mathcal{B}\left(\boldsymbol{A}^{\prime}, \boldsymbol{B}^{\prime}, \boldsymbol{C}^{\prime}, \boldsymbol{D}^{\prime}\right)
$$

where

$$
A^{\prime}=T^{-1} A T, \quad B^{\prime}=T^{-1} B, \quad C^{\prime}=C T, \quad D^{\prime}=D,
$$

for any invertible matrix $\boldsymbol{T} \in \mathbb{R}^{n \times n}$. Finally, the notation $\left.\boldsymbol{w}\right|_{T}$ denotes the restriction of $w \in \mathbb{W}^{\mathbb{N}}$ to the interval $[1, \ldots, T]$.

\section{ESTIMATION OF SYNCHRONIZATION ERRORS}

Suppose that a system described by (1) has clock synchronization errors of the form considered here. Then its dynamics change and are now described by (8) for some $s^{\star} \in \mathcal{S}$ where the entries in the matrices $\boldsymbol{A}_{s}$ and $\boldsymbol{B}_{s}$ that are not equal to 0 or 1 are not known. The problem we now solve how to identify $s^{\star}$ from the given finite trajectory

$$
\left.\left(\boldsymbol{u}^{\star}, \boldsymbol{y}^{\star}\right)\right|_{T}=\left(\left(\boldsymbol{u}^{\star}(1), \boldsymbol{y}^{\star}(1), \ldots,\left(\boldsymbol{u}^{\star}(T), \boldsymbol{y}^{\star}(T)\right),\right.\right.
$$

which is assumed to be available. The algorithm for this purpose developed below is based on the fact that a solution $\boldsymbol{x}(1)$ of

$$
\boldsymbol{Y}_{T}^{\star}=\boldsymbol{O}_{T}\left(\boldsymbol{A}_{s}, \boldsymbol{C}\right) \boldsymbol{x}(1)+\boldsymbol{T}_{T}\left(\boldsymbol{A}_{s}, \boldsymbol{B}_{s}, \boldsymbol{C}, \boldsymbol{D}\right) \boldsymbol{U}_{T}^{\star},
$$

where $\boldsymbol{Y}_{T}^{\star}$ and $\boldsymbol{U}_{T}^{\star}$ are constructed from the trajectory $\left.\left(\boldsymbol{u}^{\star}, \boldsymbol{y}^{\star}\right)\right|_{T}$ according to (13), exists for $T>n$ if, and only if, $\left.\left(\boldsymbol{u}^{\star}, \boldsymbol{y}^{\star}\right)\right|_{T}$ is a trajectory of the system $\mathcal{B}\left(\boldsymbol{A}_{s}, \boldsymbol{B}_{s}, \boldsymbol{C}, \boldsymbol{D}\right)$. Therefore, we are searching over all $s \in \mathcal{S}$ for a compatible system (16) and as a solution algorithm this is successful only if there is one $\hat{s}$ such that a solution exists and then $s^{\star}=\hat{s}$. In this case we term the synchronization error $s^{\star}$ identifiable from the trajectory $\left(\boldsymbol{u}^{\star}, \boldsymbol{y}^{\star}\right)$.

\section{A. Similar cases}

Consider (8) and a sequence $s_{1}=\left(i_{1}, i_{2}, \ldots, i_{d-1}, i_{d}\right)$ describing the switching sequence with corresponding state matrix

$$
\boldsymbol{A}_{s_{1}}=\boldsymbol{A}_{i_{d}} \boldsymbol{A}_{i_{d-1}} \cdots \boldsymbol{A}_{i_{2}} \boldsymbol{A}_{i_{1}}
$$

Assuming that all matrices $\boldsymbol{A}_{i_{j}}, j=1, \ldots, d$ are invertible, we have

$$
\begin{aligned}
\boldsymbol{A}_{i_{d}}^{-1} \boldsymbol{A}_{s_{1}} \boldsymbol{A}_{i_{d}} & =\boldsymbol{A}_{i_{d}}^{-1} \boldsymbol{A}_{i_{d}} \boldsymbol{A}_{i_{d-1}} \cdots \boldsymbol{A}_{i_{2}} \boldsymbol{A}_{i_{1}} \boldsymbol{A}_{i_{d}} \\
& =\boldsymbol{A}_{i_{d-1}} \boldsymbol{A}_{i_{d-2}} \cdots \boldsymbol{A}_{i_{1}} \boldsymbol{A}_{i_{d}} \\
& =\boldsymbol{A}_{s_{2}}
\end{aligned}
$$

and hence $\boldsymbol{A}_{s_{1}}$ is similar $\boldsymbol{A}_{s_{2}}$ corresponding to the sequence $s_{2}=\left(i_{d}, i_{1}, \ldots, i_{d-2}, i_{d-1}\right)$, where the entries in $s_{2}$ are those in $s_{1}$ shifted to the right by 1 . For $\boldsymbol{A}_{s_{2}}$

$$
\begin{aligned}
\boldsymbol{A}_{i_{d-1}}^{-1} \boldsymbol{A}_{s_{2}} \boldsymbol{A}_{i_{d-1}} & =\boldsymbol{A}_{i_{d-1}}^{-1} \boldsymbol{A}_{i_{d-1}} \boldsymbol{A}_{i_{d-2}} \cdots \boldsymbol{A}_{i_{1}} \boldsymbol{A}_{i_{d}} \boldsymbol{A}_{i_{d-1}} \\
& =\boldsymbol{A}_{i_{d-2}} \boldsymbol{A}_{i_{d-3}} \cdots \boldsymbol{A}_{i_{d}} \boldsymbol{A}_{i_{d-1}} \\
& =\boldsymbol{A}_{s_{3}}
\end{aligned}
$$

and hence this matrix is similar to $\boldsymbol{A}_{s_{3}}$ corresponding to the sequence $s_{3}=\left(i_{d-1}, i_{d}, \ldots, i_{1}, \ldots, i_{d-3}, i_{d-2}\right)$, where the entries in $s_{2}$ are those in $s_{1}$ shifted to the right by 1. Repeating the procedure until the original sequence is obtained we have that all the matrices which result are similar.

These similar cases are of special concern because the systems involved may not be distinguishable from each other based on input/output data as the following example illustrates.

Example 2: Consider the special case of (1) when $n=$ $m=3$ and

$$
\begin{array}{rlrl}
\boldsymbol{A} & =\left[\begin{array}{lll}
0.1 & 0.2 & 0.4 \\
0.2 & 0.1 & 0.3 \\
0.3 & 0.1 & 0.2
\end{array}\right], & \boldsymbol{B}=I_{3 \times 3}, \\
\boldsymbol{C}=\left[\begin{array}{lll}
1 & 0 & 0
\end{array}\right], & \boldsymbol{D}=\mathbf{0}_{1 \times 3},
\end{array}
$$

(where $I_{3 \times 3}$ is the $3 \times 3$ identity matrix and $\mathbf{0}_{1 \times 3}$ the $1 \times 3$ null matrix). Also for the three asynchronous cases described by the sequences 1$\left.) s_{1}=(\{1\},\{2\},\{3\}), 2\right) s_{2}=$ $(\{3\},\{1\},\{2\})$ and 3$) s_{3}=(\{2\},\{3\},\{1\})$ respectively, the relevant state-space model matrices are as follows.

$$
\begin{aligned}
\boldsymbol{A}_{s 1} & =\left[\begin{array}{ccc}
0.1 & 0.2 & 0.4 \\
0.02 & 0.14 & 0.38 \\
0.032 & 0.074 & 0.358
\end{array}\right], \\
\boldsymbol{B}_{s 1} & =\left[\begin{array}{ccc}
1 & 0 & 0 \\
0.2 & 1 & 0 \\
0.32 & 0.1 & 1
\end{array}\right], \\
\text { 2) } \boldsymbol{A}_{s 2} & =\left[\begin{array}{ccc}
0.22 & 0.24 & 0.08 \\
0.134 & 0.178 & 0.076 \\
0.3 & 0.1 & 0.2
\end{array}\right], \\
\boldsymbol{B}_{s 2} & =\left[\begin{array}{ccc}
1 & 0 & 0.4 \\
0.2 & 1 & 0.38 \\
0 & 0 & 1
\end{array}\right],
\end{aligned}
$$

$$
\begin{aligned}
\boldsymbol{A}_{s 3} & =\left[\begin{array}{ccc}
0.268 & 0.024 & 0.152 \\
0.2 & 0.1 & 0.3 \\
0.32 & 0.01 & 0.23
\end{array}\right], \\
\boldsymbol{B}_{s 3} & =\left[\begin{array}{ccc}
1 & 0.24 & 0.4 \\
0 & 1 & 0 \\
0 & 0.1 & 1
\end{array}\right]
\end{aligned}
$$


For this example, the initial conditions

$$
\begin{aligned}
\text { 1) } \boldsymbol{x}_{0,1}=\left[\begin{array}{l}
10 \\
10 \\
10
\end{array}\right], \text { 2) } \boldsymbol{x}_{0,2}=\left[\begin{array}{c}
10 \\
10 \\
0
\end{array}\right], \\
\text { 3) } \boldsymbol{x}_{0,3}=\left[\begin{array}{c}
10 \\
23.333 \\
0
\end{array}\right],
\end{aligned}
$$

and the input

$$
\boldsymbol{u}(k)=\left[\begin{array}{lll}
1 & 1 & 1
\end{array}\right]^{T}, k=1,2, \ldots
$$

produce the same output in all three cases. Hence (16) holds for all these systems and therefore they are not distinguishable from the trajectory $(u, y)$, where $y$ is the response of each of them to the input sequence (17).

\section{IDENTIFIABILITY OF CLOCK SYNCHRONIZATION ERRORS}

Let $\mathcal{B}_{s}$ denote the behavior of the system with clock synchronization $s$, that is,

$$
\mathcal{B}_{s}=\mathcal{B}\left(\boldsymbol{A}_{s}, \boldsymbol{B}_{s}, \boldsymbol{C}, \boldsymbol{D}\right) \text {. }
$$

Then solution to (16) exists if, and only if, $\left.\left(\boldsymbol{u}^{\star}, \boldsymbol{y}^{\star}\right)\right|_{T}$ belongs to $\mathcal{B}_{s}$, that is,

$$
\exists(\boldsymbol{u}, \boldsymbol{y}) \in \mathcal{B}_{s},\left.\quad(\boldsymbol{u}, \boldsymbol{y})\right|_{T}=\left.\left(\boldsymbol{u}^{\star}, \boldsymbol{y}^{\star}\right)\right|_{T},
$$

Hence there is only one $\hat{s} \in \mathcal{S}$ such that (16) has a solution when the following hold.

1) There is only one system $\mathcal{B}$ with $m$ inputs and $n$ state variables. containing the trajectory $\left(\boldsymbol{u}^{\star}, \boldsymbol{y}^{\star}\right)$.

2) The map $s \mapsto B_{s}$ is injective, that is, $\forall s_{1}, s_{2} \in \mathcal{S}$, $s_{1} \neq s_{2} \Longrightarrow \mathcal{B}_{s_{1}} \neq \mathcal{B}_{s_{2}}$.

\section{A. Conditions on the trajectory}

As illustrated by Example 2, the trajectory $\left.\left(\boldsymbol{u}^{\star}, \boldsymbol{y}^{\star}\right)\right|_{T}$ can be obtained from more than one behavior with $m$ inputs and $n$ state variables. In order to guarantee that there is only one such behavior, conditions on the trajectory must be imposed. Following [8] assume that

a) the system with synchronization errors is controllable,

b) the trajectory $\left.\left(\boldsymbol{u}^{\star}, \boldsymbol{y}^{\star}\right)\right|_{T}$ is exact (noise free),

c) $u$ is persistently exciting of order $k=n+l+1$, where

$l$ is the order of the lag [9], that is, the matrix

$$
\mathcal{U}=\left[\begin{array}{cccc}
\boldsymbol{u}^{\star}(1) & \boldsymbol{u}^{\star}(2) & \ldots & \boldsymbol{u}^{\star}(T-k+1) \\
\boldsymbol{u}^{\star}(2) & \boldsymbol{u}^{\star}(3) & \ldots & \boldsymbol{u}^{\star}(T-k+2) \\
\ldots & \ldots & \ldots & \ldots \\
\boldsymbol{u}^{\star}(k) & \boldsymbol{u}^{\star}(k+1) & \ldots & \boldsymbol{u}^{\star}(T)
\end{array}\right]
$$

has full row rank.

Then the matrices defining the state-space model of the system with synchronization errors can be determined from the trajectory $\left(\boldsymbol{u}^{\star}, \boldsymbol{y}^{\star}\right)$. In other words. this is a sufficient condition to guarantee that the obtained trajectory belongs to only one system with $m$ inputs and $n$ state variables. This will eliminate the problem with similar cases (Section III-A).

\section{B. Conditions for the injectivity of the map $s \mapsto \mathcal{B}_{s}$}

In order to determine the unknown clock synchronization error $s^{\star}$ we require that

$$
s_{1} \neq s_{2} \quad \Longrightarrow \quad \mathcal{B}_{s_{1}} \neq \mathcal{B}_{s_{2}}, \forall s_{1}, s_{2} \in \mathcal{S},
$$

or, equivalently, for all $s_{1}, s_{2} \in \mathcal{S}$ such that $s_{1} \neq s_{2}$ there is no invertible matrix $\boldsymbol{T} \in \mathbb{R}^{n \times n}$ such that

$$
\boldsymbol{T}^{-1} \boldsymbol{A}_{s_{1}} \boldsymbol{T}=\boldsymbol{A}_{s_{2}}, \quad \boldsymbol{T}^{-1} \boldsymbol{B}_{s_{1}}=\boldsymbol{B}_{s_{2}}, \quad \boldsymbol{C T}=\boldsymbol{C},
$$

that is,

$$
\boldsymbol{A}_{s_{1}} \boldsymbol{T}=\boldsymbol{T} \boldsymbol{A}_{s_{2}}, \quad \boldsymbol{B}_{s_{1}}=\boldsymbol{T} \boldsymbol{B}_{s_{2}}, \quad \boldsymbol{C T}=\boldsymbol{C} .
$$

The condition (21) is a linear system of equations in the unknown transformation matrix $\boldsymbol{T}$ and in order to check injectivity of the map $s \mapsto \mathcal{B}_{S} O\left(\operatorname{card}(S)^{2}\right)$ systems of this form have to be solved. This is impractical for realistic size problems since $\operatorname{card}(S)$ for a system with $n$ state variables can be approximated by $\sqrt{2}^{n} n$ !. Consequently we have to search over all $s \in \mathcal{S}$ for a solution of (16). Note, however, that a simple necessary condition for injectivity exists as detailed next.

Let $a_{p q}, 1 \leq p, q \leq n$, denote the entries in the matrix $\boldsymbol{A}$ of (1) and suppose that at least one of them is zero. Also let the sequence $s_{1}=\left(i_{1}, \ldots, i_{j}, i_{j+1} \ldots, i_{d}\right)$, with $i_{j}=\{q\}$ and $i_{j+1}=\{p\}$, describe the switching event pattern. Hence

$$
\begin{aligned}
\boldsymbol{x}^{j}(k) & =\boldsymbol{A}_{\{q\}} \boldsymbol{x}^{j-1}(k)+\boldsymbol{B}_{\{q\}} \boldsymbol{u}(k), \\
\boldsymbol{x}^{j+1}(k) & =\boldsymbol{A}_{\{p\}} \boldsymbol{x}^{j}(k)+\boldsymbol{B}_{\{p\}} \boldsymbol{u}(k),
\end{aligned}
$$

and therefore

$\boldsymbol{x}^{j+1}(k)=\boldsymbol{A}_{\{p\}} \boldsymbol{A}_{\{q\}} \boldsymbol{x}^{j-1}(k)+\left(\boldsymbol{B}_{\{p\}}+\boldsymbol{A}_{\{p\}} \boldsymbol{B}_{\{q\}}\right) \boldsymbol{u}(k)$.

Here the updating in $p$ occurs after that in $q$ but the computations for the former do not involve values for the latter since by assumption $a_{p q}=0$. Equivalently, nothing changes if these variables update simultaneously since

$$
\boldsymbol{A}_{\{p\}} \boldsymbol{A}_{\{q\}}=\boldsymbol{A}_{\{p, q\}},
$$

and

$$
\boldsymbol{A}_{\{p\}} \boldsymbol{B}_{\{q\}}=\boldsymbol{B}_{\{q\}} \Rightarrow\left(\boldsymbol{B}_{\{p\}}+\boldsymbol{A}_{\{p\}} \boldsymbol{B}_{\{q\}}\right)=\boldsymbol{B}_{\{p, q\}},
$$

where these last two facts follow immediately from (6) and (7) and the assumption that $a_{p q}=0$. Hence we can write (22) as

$$
\boldsymbol{x}^{j+1}(k)=\boldsymbol{A}_{\{p, q\}} \boldsymbol{x}^{j-1}(k)+\boldsymbol{B}_{\{p, q\}} \boldsymbol{u}(k),
$$

and if we consider the sequence of length $d-1$

$$
s_{2}=\left(i_{1}, \ldots, i_{j-1}, i_{j} \cup i_{j+1}, i_{j+2}, \ldots, i_{d}\right),
$$

then both $s_{1}$ and $s_{2}$ yield the same state-space model. Therefore if there is a zero entry in the matrix $\boldsymbol{A}$, the map $s \mapsto \mathcal{B}_{s}$ is not injective. By contraposition we have that nonzero entries in the system matrix is a necessary condition for the injectivity of the map $s \mapsto \mathcal{B}_{s}$. 
Example 3: Consider the single-input single-output system

$$
\begin{aligned}
\boldsymbol{x}(k+1) & =\left[\begin{array}{lll}
0.1 & 0.2 & 0.4 \\
0.2 & 0.1 & 0.2 \\
0.3 & 0.6 & 0.6
\end{array}\right] \boldsymbol{x}(k) \\
& +\left[\begin{array}{l}
0.2 \\
0.4 \\
0.3
\end{array}\right] u(k), \\
y(k) & =\left[\begin{array}{lll}
1 & 0 & 0
\end{array}\right] \boldsymbol{x}(k) .
\end{aligned}
$$

The matrix $\boldsymbol{A}$ in this case has no zero entries and hence the necessary condition is satisfied. Moreover, using (2)(4) we know that there are 13 state-space models that could be the model of the system dynamics in the presence of synchronization errors of the form considered in this work. Also we can compute the matrices of the state-space model (8) describing the dynamics after all switching events have occurred.

In a physical application, the next step would be to obtain the response of the system with synchronization errors (assumed to be controllable and hence a) above is satisfied) to an input sequence that satisfying conditions b) and c) above. Here, in order to demonstrate that the algorithm can correctly identify the synchronization error sequence, suppose that one of the 13 state-space models is given by

$$
\begin{aligned}
\boldsymbol{x}(k+1) & =\left[\begin{array}{ccc}
0.1 & 0.2 & 0.4 \\
0.02 & 0.14 & 0.28 \\
0.042 & 0.144 & 0.888
\end{array}\right] \boldsymbol{x}(k) \\
& +\left[\begin{array}{c}
0.2 \\
0.44 \\
0.624
\end{array}\right] u(k), \\
y(k) & =\left[\begin{array}{lll}
1 & 0 & 0
\end{array}\right] \boldsymbol{x}(k)
\end{aligned}
$$

and the next stage is to obtain the output of this last system in response to an input sequence that satisfies (19). By the identifiability condition of [7], the number of input values should be $T \geq 4 n+1=13$ and one input sequence that satisfies this condition is (recall (13)) $\boldsymbol{U}_{13}=\left[\begin{array}{ll}X_{1} & X_{2}\end{array}\right]^{T}$, where

$$
\begin{aligned}
& X_{1}=\left[\begin{array}{llllllllll}
1, & 2, & 3, & 4, & 5, & 6, & 7, & 8, & 7, & 8
\end{array}\right] \\
& X_{2}=\left[\begin{array}{lll}
8 & 7 & 8
\end{array}\right]
\end{aligned}
$$

On computing the response of (23) to this input (with a free choice of state initial conditions), we have from (16) that $x(1)$ exists in this case only when

$$
s^{*}=(\{1\},\{2\},\{3\}) \text {. }
$$

Finally, applying this synchronization error to the system state-space model with no synchronization errors we generate precisely the state-space model (23). Hence the identification algorithm is verified for this particular example.

\section{EFFECTS OF GUASSIAN MEASUREMENT NOISE}

In this section we assume that the collected output values from the system with synchronization errors is corrupted by Gaussian measurement noise, where if the signal to noise ratio is relatively small a first step could be to again apply the technique developed in this paper to estimate the synchronization errors.

Assume that the number of observations exceeds the state vector dimension. Hence the resulting system of equations (16) is overdetermined and can be written in the form

$$
\begin{aligned}
\boldsymbol{O}_{T}^{T}\left(\boldsymbol{A}_{s}, \boldsymbol{C}\right) \boldsymbol{O}_{T}\left(\boldsymbol{A}_{s}, \boldsymbol{C}\right) \boldsymbol{x}(1)=\boldsymbol{O}_{T}^{T}\left(\boldsymbol{A}_{s}, \boldsymbol{C}\right) \boldsymbol{Y}_{T}^{\star} \\
-\boldsymbol{O}_{T}^{T}\left(\boldsymbol{A}_{s}, \boldsymbol{C}\right) \boldsymbol{T}_{T}\left(\boldsymbol{A}_{s}, \boldsymbol{B}_{s}, \boldsymbol{C}, \boldsymbol{D}\right) \boldsymbol{U}_{T}^{\star} .
\end{aligned}
$$

Also this system of equations can be solved, see [10], without computing a matrix inverse to obtain the solution $\boldsymbol{x}^{\star}(1)$ that minimizes the norm

$$
\left\|\boldsymbol{Y}_{T}^{\star}-\boldsymbol{O}_{T}\left(\boldsymbol{A}_{s}, \boldsymbol{C}\right) \boldsymbol{x}^{\star}(1)-\boldsymbol{T}_{T}\left(\boldsymbol{A}_{s}, \boldsymbol{B}_{s}, \boldsymbol{C}, \boldsymbol{D}\right) \boldsymbol{U}_{T}^{\star}\right\|_{2}
$$

If the measurements are completely noise free then the only candidate solution that will return a zero solution is the correct one.

In the case when the output signal is corrupted by noise, the change in the norms will not exceed the norm of the noise signal. Hence if the norm of the noise signal is relatively small then we may expect that the norm (25) for the correct system will remain smaller than the rest. However, the level of acceptable noise depends on the system under consideration and also increasing the number of observations is not helpful because it increases the norm of the noise signal and this may cause difficulties when comparing norms.

To apply this method, we first compute the solutions of (24) for all candidate systems as in the noise free case detailed in the previous section. Then we compare the results norms in (25) for all these and if one is significantly smaller than others this is taken as the solution.

Example 4: Consider the case when

$$
\begin{aligned}
\boldsymbol{x}(k+1) & =\left[\begin{array}{ccc}
-0.3778 & -0.6304 & -0.1223 \\
0.8468 & 0.8098 & -0.7778 \\
-0.1396 & 0.9595 & -0.4839
\end{array}\right] \boldsymbol{x}(k) \\
& +\left[\begin{array}{lll}
1 & 0 & 0 \\
0 & 1 & 0 \\
0 & 0 & 1
\end{array}\right] u(k), \\
y(k) & =\left[\begin{array}{lll}
1 & 0 & 0
\end{array}\right] \boldsymbol{x}(k),
\end{aligned}
$$

and suppose that the clock synchronization error is specified by $(\{1,2\},\{3\})$ resulting in the state-space model

$$
\begin{aligned}
& \boldsymbol{x}(k+1)=\left[\begin{array}{ccc}
-0.3778 & -0.6304 & -0.1223 \\
0.8468 & 0.8098 & -0.7778 \\
0.8652 & 0.8650 & -1.2131
\end{array}\right] \boldsymbol{x}(k) \\
&+ {\left[\begin{array}{ccc}
1.0000 & 0 & 0 \\
0 & 1.0000 & 0 \\
-0.1396 & 0.9595 & 1.0000
\end{array}\right] u(k), } \\
& y(k)=\left[\begin{array}{lll}
1 & 0 & 0
\end{array}\right] \boldsymbol{x}(k) .
\end{aligned}
$$

and take the initial state $\boldsymbol{x}(1)=[10,10,10]^{T}$. Suppose also that a random input generated the response shown in Fig. 1. Comparison of norms of (25) in this case is illustrated in Fig. 4 and by inspection it is system 7 which has synchronization error $(\{1,2\},\{3\})$. Hence the synchronization error has been correctly determined. 


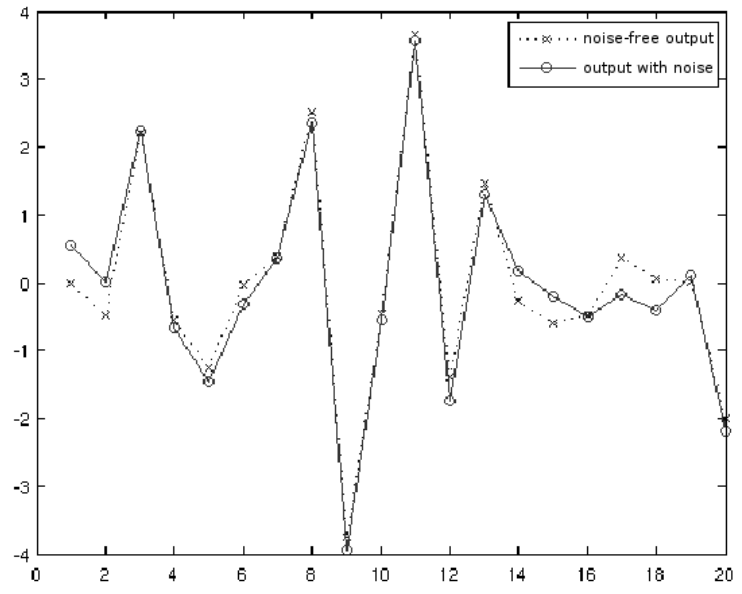

Fig. 1. System output in response to a random input with synchronization error present.

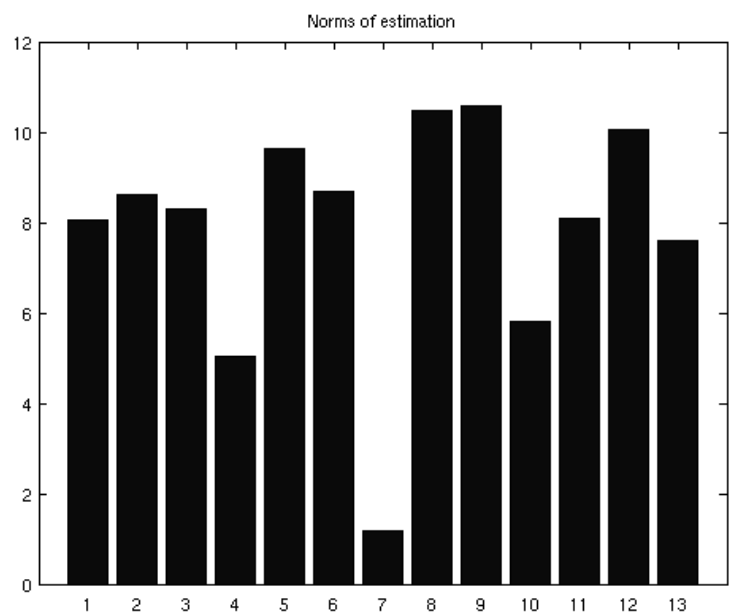

Fig. 2. Comparison of the norms of the candidate systems.

\section{CONCLUSIONS}

A method has been developed for identification of synchronization error from input output data of an asynchronously operating linear system. The resulting algorithm requires only the solution of a set of linear equations and is easy to implement. Further research is required to improve the computational feasibility of the identifiability conditions.

\section{REFERENCES}

[1] C. Lorand and P. Bauer, "Distributed discrete time systems with synchronization errors: Models and stability," IEEE Trans on Circ \& Syst., CAS-II Express Briefs, vol. 52, no. 4, pp. 208-213, April 2005.

[2] - "On synchronization errors in networked feedback systems," IEEE Trans. on Circuits and Systems CAS-I Regular Papers, vol. 53, no. 10 , October 2006.

[3] — - "Clock synchronization errors in circuits: Models, stability and fault detection," IEEE Transaction on Circ. and Syst., CAS-I Regular papers, vol. 53, no. 10, 2006.
[4] A. F. Kleptzyn, V. S. Kozyakin, M. Krasnoselskii, and N. Kuznetsov, "Effects of small synchronization errors on complex systems," Avtomatika o Telemekhanika. Translated in: Aut. and Remote control, pp. 1014-1018, 1984.

[5] Y. F. Su, A. Bhaya, E. Kaszkurewicz, and V. S. Kozyakin, "Further results on stability of asynchronous discrete time linear system," Proc. IEEE Conf. on CDC San Diego, pp. 915-920, 1997.

[6] C. Lorand, "A theory of synchronization errors," Ph.D Thesis, University of Notre Dame, 2004, Notre Dame, IN.

[7] J. C. Willems, "The behavioral approach to open and interconnected systems," Control Systems Magazine, vol. 27, pp. 46-99, 2007.

[8] J. Willems, P. Rapisarda, I. Markovsky, and B. de Moor, "A note on persistency of excitation," Systems and Control Letters, vol. 54, pp. 325-329, 2005

[9] J. C. Willems, "Paradigms and puzzles in the theory of dynamical systems," IEEE Trans. Automat. Control, vol. 36, no. 3, pp. 259-294, 1991.

[10] G. H. Golub and C. F. van Loan, "Matrix computations, third edition," The Johns Hopkins Univesrity Press, Maryland, 1996 